\title{
Preparation of 9-Substituted Pyridine-Stretched Adenines and Hypoxanthine
}

\author{
Julia A. Crawford, ${ }^{\text {a }}$ William Fraser, ${ }^{\mathrm{b}}$ Christopher A. Ramsden ${ }^{\mathrm{a} *}$ \\ ${ }^{\text {a }}$ Lennard-Jones Laboratories, School of Physical and Geographical Sciences, Keele University, Keele, Staffordshire, UK ST5 5BG \\ ${ }^{\mathrm{b}}$ Pharmaceutical Sciences Research Institute, Aston University, Aston Triangle, Birmingham, B4 7ET, UK \\ Fax: 01782712378 \\ E-mail: c.a.ramsden@chem.keele.ac.uk \\ Received: [Draft of 09/10/08]
}

Abstract: Please overwrite this text with a written abstract, which should summarize the results and conclusions of the research performed.

Key words: imidazoles, amines, lin-pyridoadenines, soft electrophile, tricyclic heterocycle.

Several groups have investigated modification of purine nucleosides by insertion of a third ring between the imidazole and pyrimidine fragments. The earliest investigation was by Leonard and coworkers who described the synthesis of the benzene-stretched adenosine analogue 1. ${ }^{1-4}$ Later the 2ôdeoxy derivative 3 was prepared. ${ }^{5}$ Subsequently other groups have prepared tricyclic analogues ${ }^{6-8}$ and aspects of this work have been reviewed. 9 For some time we have been interested in preparing pyridine-stretched purine derivatives and have previously described the adenosine analogue 2 and the corresponding inosine analogue. ${ }^{10}$ More recently we prepared the $2 \hat{o}$ deoxy analogues of adenosine 4 and inosine: ${ }^{11}$ these have been incorporated into oligonucleotides and their duplex-forming properties investigated. ${ }^{12}$ With the objective of providing access to a wider range of pyridine-stretched purine derivatives, we have investigated the preparation of 9-substituted derivatives of the general types 5 and $6(\mathrm{R}$ I H) and now report the results of these studies.

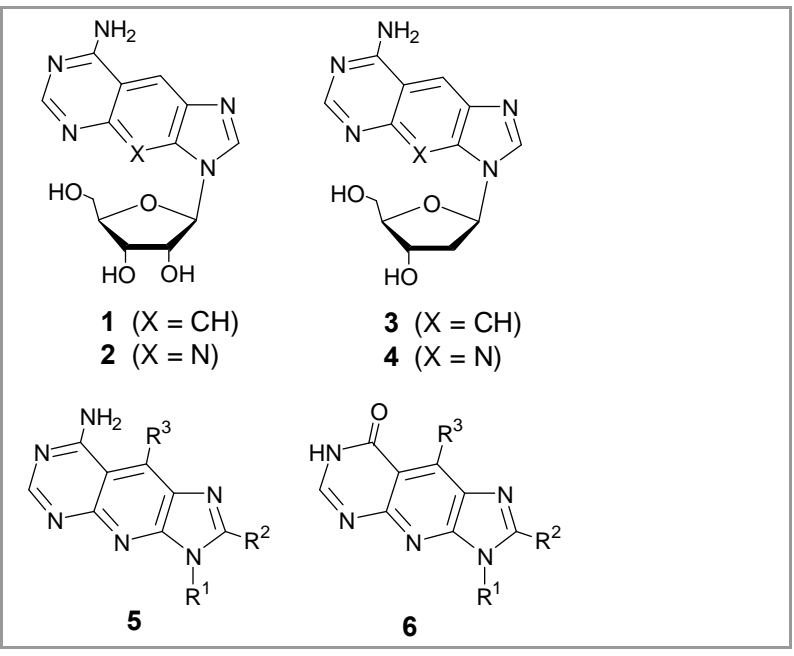

Figure 1

Two features of pyridine-stretched nucleosides may be advantageous: (i) the presence of the nitrogen atom in position 4 may enhance the stability of triplexforming oligonucleotides (TFOs) targeted to DNA; (ii) their preparation via addition-elimination reactions of 5-aminoimidazoles may make them more easily accessible than the benzene-stretched derivatives. The 9-unsubstituted derivatives $\mathbf{2}$ and $\mathbf{4}$ were prepared via reaction of ethoxymethylene malononitrile [EMMN: $\left.(\mathrm{NC})_{2} \mathrm{C}=\mathrm{CHOEt}\right]$ with the appropriate 5aminoimidazoles $\mathbf{8}$, which were obtained by reduction of the 5-nitroimidazoles $7 .^{10,11}$ The preparation of the 9-substituted derivatives 5 and $6(\mathrm{R} \mid \mathrm{H})$ therefore requires a $\mathrm{C}$-substituted analogue of EMMN that reacts selectively at the C-4 position of 5aminoimidazoles $\mathbf{8}$.

\begin{tabular}{|ll} 
& \\
$7(\mathrm{Y}=\mathrm{O})$ & $9 \mathrm{Z}=\mathrm{SMe}$ \\
$8(\mathrm{Y}=\mathrm{H})$ & $10 \mathrm{Z}=\mathrm{NHnPr}$ \\
& $11 \mathrm{Z}=\mathrm{NHPh}$ \\
\hline
\end{tabular}

Figure 2

In an earlier study, ${ }^{13,14}$ we showed that 5aminoimidazoles $^{15}$ can react as either $\mathrm{N}$ - or Cnucleophiles and demonstrated a correlation between the position of reaction and the softness of the electrophile, measured by the AM1 calculated LUMO energy. Thus, EMMN [(NC) $\left.{ }_{2} \mathrm{C}=\mathrm{CHOEt}\right]$ (LUMO $0.62 \mathrm{eV}$ ) gives exclusively $\mathrm{C}$-adducts whereas diethyl ethoxymethylenemalonate

[EMME: $\left(\mathrm{EtO}_{2} \mathrm{C}\right)_{2} \mathrm{C}=\mathrm{CHOEt}$ (LUMO $-0.27 \mathrm{eV}$ ) gives exclusively $\mathrm{N}$-adducts. The general conclusion of our studies of a range of electrophiles was that reagents with a calculated (AM1) LUMO energy $<0.5 \mathrm{eV}$ react predominantly at the C-4 position of 5aminoimidazoles. $^{14} \mathrm{~A}$ C-substituted analogue of EMMN with a LUMO energy $<0.5 \mathrm{eV}$ was therefore required in order to produce precursors of the 9substituted derivatives 5 and $\mathbf{6}$. Table 1 shows the AM1 calculated frontier orbital energies of selected electrophiles.

From Table 1 it is clear that introduction of a second ethoxy substituent into EMME (Entries 2 and 4) will increase the LUMO energy and probably favour Nadduct formation. However, replacement of the ethoxy group by a methylsulfanyl substituent (Entries 4 and 7) significantly reduces the LUMO energy and intro- 
duction of a second methylsulfanyl group (Entry 8) leads to an electrophile with an exceptionally low energy LUMO. We therefore anticipated that 2-(bismethylsulfanylmethylene) malononitrile 9 $\left[(\mathrm{NC})_{2} \mathrm{C}=\mathrm{C}(\mathrm{SMe})_{2}\right]$ would give $\mathrm{C}$-adducts with 5aminoimidazoles and thus provide access to 9methylsulfanyl derivatives that can be further manipulated. Although we have not previously worked with the reagent $\mathbf{9}$, it is readily prepared in good yield by reaction of malononitrile with carbon disulfide and methyl iodide in the presence of potassium fluoride. ${ }^{16}$ The LUMO energy lowering effect of an alkylsulfanyl substituent appears to be general: a similar effect is observed for the corresponding diesters (Entries 1 and 3).

Table 1 AM1 calculated frontier orbital energies of electrophiles

\begin{tabular}{clll}
\hline Entry & Electrophile & $\begin{array}{l}\text { LUMO } \\
(\mathbf{e V})\end{array}$ & $\begin{array}{l}\text { HOMO } \\
(\mathbf{e V})\end{array}$ \\
\hline $\mathbf{1}$ & $\left(\mathrm{EtO}{ }_{2} \mathrm{C}\right)_{2} \mathrm{C}=\mathrm{CH} . \mathrm{OEt}$ & -0.27 & -10.15 \\
$\mathbf{2}$ & $(\mathrm{NC})_{2} \mathrm{C}=\mathrm{C}(\mathrm{OEt})_{2}$ & -0.29 & -9.53 \\
$\mathbf{3}$ & $\left(\mathrm{EtO}{ }_{2} \mathrm{C}\right)_{2} \mathrm{C}=\mathrm{CH} . \mathrm{SMe}$ & -0.60 & -9.05 \\
$\mathbf{4}$ & $(\mathrm{NC})_{2} \mathrm{C}=\mathrm{CH} . \mathrm{OEt}$ & -0.62 & -10.05 \\
$\mathbf{5}$ & $(\mathrm{NC})_{2} \mathrm{C}=\mathrm{C}(\mathrm{SMe}) \mathrm{NH}^{\mathrm{n}} \mathrm{Pr}$ & -0.71 & -8.93 \\
$\mathbf{6}$ & $(\mathrm{NC})_{2} \mathrm{C}=\mathrm{C}(\mathrm{SMe}) \mathrm{NHPh}$ & -0.90 & -8.87 \\
$\mathbf{7}$ & $(\mathrm{NC})_{2} \mathrm{C}=\mathrm{CH} . \mathrm{SMe}$ & -0.95 & -9.11 \\
$\mathbf{8}$ & $(\mathrm{NC})_{2} \mathrm{C}=\mathrm{C}(\mathrm{SMe})_{2}$ & -1.31 & -8.91 \\
\hline
\end{tabular}

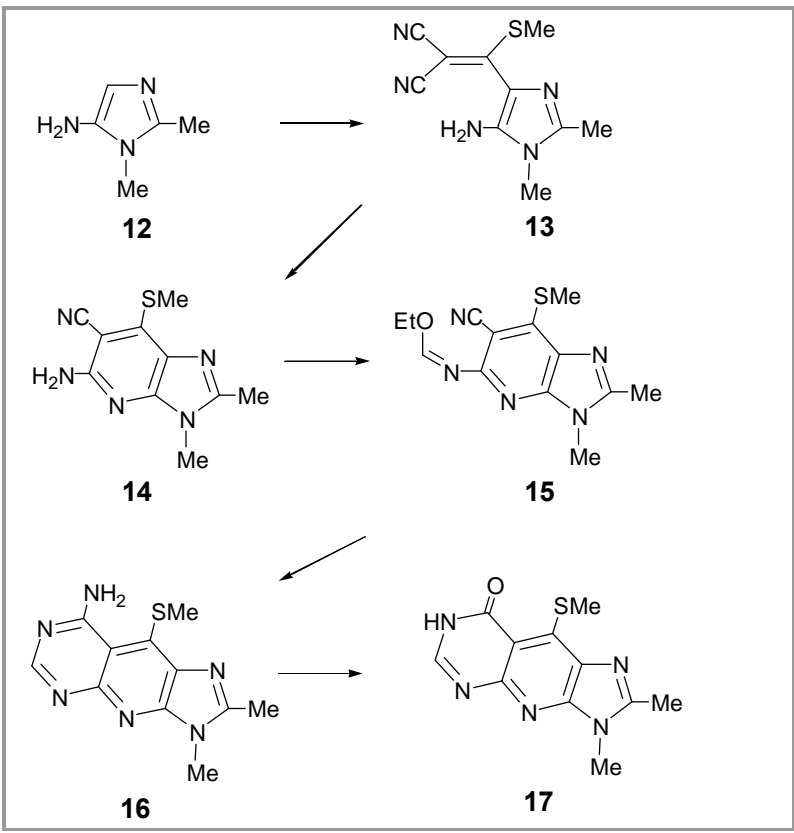

Scheme 1

In these studies we have used 5-amino-1,2dimethylimidazole $\mathbf{1 2}$ as a model compound. A solu- tion of the amine 12 in THF was generated by catalytic reduction $\left(\mathrm{H}_{2} / \mathrm{Pd} / \mathrm{C}\right)$ of 1,2-dimethyl-5nitroimidazole $7\left(\mathrm{R}^{1}=\mathrm{R}^{2}=\mathrm{Me}\right)$. The amine solution was filtered through celite into a flask containing a five-fold excess of 2-(bis-methylsulfanylmethylene) malononitrile 9 and the mixture stirred at $50{ }^{\circ} \mathrm{C}$ overnight. Workup gave a yellow-orange solid that was identified as the $\mathrm{C}$-adduct 13. The yield based on nitoimidazole starting material was $47 \%$. No other products were identified in the reaction mixture. An analytically pure sample (mp $197-8{ }^{\circ} \mathrm{C}$ ) was prepared by flash chromatography and recrystallisation from EtOAc/MeOH. The structure 13 was fully supported by its spectroscopic properties. In particular the ${ }^{1} \mathrm{H}$ NMR spectrum showed a broad singlet at $\delta 7.05$ corresponding to the $\mathrm{NH}_{2}$ group and the absence of an imidazole ring proton, which would be characteristic of the isomeric $\mathrm{N}$-adduct. The constitution $\mathrm{C}_{10} \mathrm{H}_{11} \mathrm{~N}_{5} \mathrm{~S}$ was confirmed by mass spectrometry and elemental analysis.

Treatment of a methanol solution of the C-adduct $\mathbf{1 3}$ with aqueous $\mathrm{NaOH}$ at $60{ }^{\circ} \mathrm{C}$ resulted in cyclisation to the imidazo[4,5-b]pyridine $14\left(\mathrm{mp} 300{ }^{\circ} \mathrm{C}, 86 \%\right)$. Subsequent treatment with hot triethyl orthoformate gave the ethyl imidate 15 (mp 197-200 ${ }^{\circ} \mathrm{C}, 87 \%$ ) which upon reaction with hot ethanolic ammonia gave the tricyclic amine $16\left(\mathrm{mp} 252-5{ }^{\circ} \mathrm{C}, 85 \%\right)$. The properties of the amine $\mathbf{1 6}$ were fully in accord with the proposed structure. The ${ }^{1} \mathrm{H}$ NMR spectrum showed C$\mathrm{Me}, \mathrm{S}-\mathrm{Me}$ and N-Me singlets, at $\delta 2.66,2.94$ and 3.75 respectively, together with a broad $\mathrm{NH}_{2}$ signal $(\delta 8.32)$ and a single ring proton $(\delta 8.40)$. The mass spectrum showed a strong molecular ion $(\mathrm{m} / \mathrm{z} 260)$. Compound $\mathbf{1 6}$ is an example of a 9-substituted pyridine-stretched adenine derivative and the route shown in Scheme 1 employing the soft bis-methylsulfanyl electrophile 9, therefore establishes a viable route to derivatives of this type. Treatment of compound 16 with hot $2 \mathrm{M} \mathrm{HCl}$ $(2 \mathrm{~h})$ gave the pyridine-stretched hypoxanthine derivative $17\left(\mathrm{mp}>350{ }^{\circ} \mathrm{C}, 74 \%\right)$ which was fully characterised.

Reaction of the ethyl imidate $\mathbf{1 5}$ with n-propylamine under various conditions gave only the amidine $\mathbf{1 8}$ and not the anticipated tricyclic $N$-n-propyl derivative 19. All attempts at cyclisation were unsuccessful, although similar cyclisations of 9-unsubstituted derivatives have been reported. ${ }^{15}$

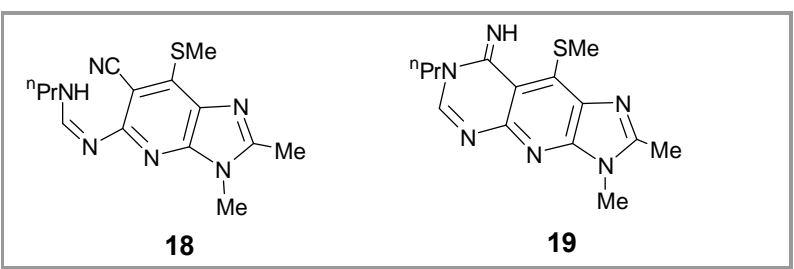

Figure 3

Inspection of Table 1 reveals that amino substituted electrophiles (Entries 5 and 6) also have a low energy 
LUMO and might be expected to react selectively to give $\mathrm{C}$-adducts. In principle these reagents could provide a direct route to 9-amino derivatives, e.g. 5 and 6 $\left(\mathrm{R}^{3}=\mathrm{NHR}\right)$. We therefore investigated the reactions of the reagents $\mathbf{1 0}$ and $\mathbf{1 1}$ with the aminoimidazole $\mathbf{1 2}$ but in neither case was any reaction detected under a variety of conditions. As might be expected, the nitrogen lone pair appears to reduce the reactivity of the electrophile and although the LUMO energy is an indicator of regioselectivity it is not a measure of reactivity. To prepare 9-amino derivatives we next investigated methods of modifying the 9methylsulfanyl substituent.

Reaction of compound $\mathbf{1 6}$ with n-propylamine did not result in substitution of the thiomethyl group by the amine. It was therefore decided to oxidize the thioether to the methyl sulfone which is a better leaving group. To avoid oxidation of the 8-amino group, Nprotected derivatives were prepared. Reaction with either acetic anhydride or acetic anhydride/ pyridine under various conditions gave the $N, N$-diacetyl derivative $20\left(\mathrm{mp} 178-183{ }^{\circ} \mathrm{C}\right)$. In one experiment a very low yield of material (mp $218-220{ }^{\circ} \mathrm{C}$ ) that appeared to be the mono-acetyl derivative was obtained but attempts to repeat this by variation of method or work-up were unsuccessful. Attempts to remove one acetyl group using calcium carbonate in $\mathrm{MeOH} / \mathrm{H}_{2} \mathrm{O}^{99}$ resulted in removal of both acetyl groups. We therefore decided to carry out further work on the diacetyl derivative 20. The 8-amino group was also protected as the t-butoxycarbonyl (t-Boc) derivative $\mathbf{2 1}$ ( $\mathrm{mp} 167$ ${ }^{\circ} \mathrm{C}(\mathrm{d}): 83 \%$ ) by reaction with di-t-butyl dicarbonate in 1,4-dioxane $\left(6 \mathrm{~h}\right.$ at $\left.120^{\circ} \mathrm{C}\right)$.

Oxidation of the $N, N$-diacetyl derivative 20 with one equivalent of 3-chloroperoxybenzoic acid (MCPBA) in $\mathrm{CH}_{2} \mathrm{Cl}_{2}$ at $0{ }^{\circ} \mathrm{C}$ gave a poor yield $(11 \%)$ of the sulfoxide 22. A similar procedure gave a better yield (63\%) of the sulfoxide 23 . Both sulfoxides were fully characterized and showed the expected spectroscopic properties. Oxidation using two equivalents of MCPBA gave the sulfones 24 (mp 185-6 ${ }^{\circ} \mathrm{C}, 67 \%$ ) and 25 (mp $\left.100{ }^{\circ} \mathrm{C}(\mathrm{d}), 65 \%\right)$, respectively. Further studies were carried out using the t-Boc protected sulfone 25, which was obtained in greater overall yield from the amine $\mathbf{1 6}$.

To demonstrate nucleophilic substitution of the methyl sulfonyl substituent we initially reacted compound $\mathbf{2 5}$ with sodium ethoxide in ethanol at room temperature. A single product was formed and, after chromatographic isolation in $24 \%$ yield, was identified as the 9ethoxy derivative (mp $\left.204{ }^{\circ} \mathrm{C}(\mathrm{d})\right)$. ${ }^{1} \mathrm{H}$ NMR spectroscopy clearly showed the product contained an ethyl substituent ( $\delta 1.67$ and 5.34) and the absence of a methyl sulfone signal $(\delta \sim 3.9)$. Excellent yields of substitution products were obtained, without chromatography, when the sulfone $\mathbf{2 5}$ was reacted with alkylamines. With n-propylamine in $\mathrm{CH}_{2} \mathrm{Cl}_{2}$ at room temperature $(2 \mathrm{~h})$ a $98 \%$ yield of the secondary amine 27 (mp $218{ }^{\circ} \mathrm{C}(\mathrm{d})$ ) was obtained. A similar procedure gave the n-butyl derivative $\mathbf{2 8}\left(\mathrm{mp} 220{ }^{\circ} \mathrm{C}(\mathrm{d})\right)$ in $93 \%$ yield. The use of 9-sulfones, e.g. 25, therefore provides access to 8,9-diamino derivatives in the pyridine-stretched purine series.

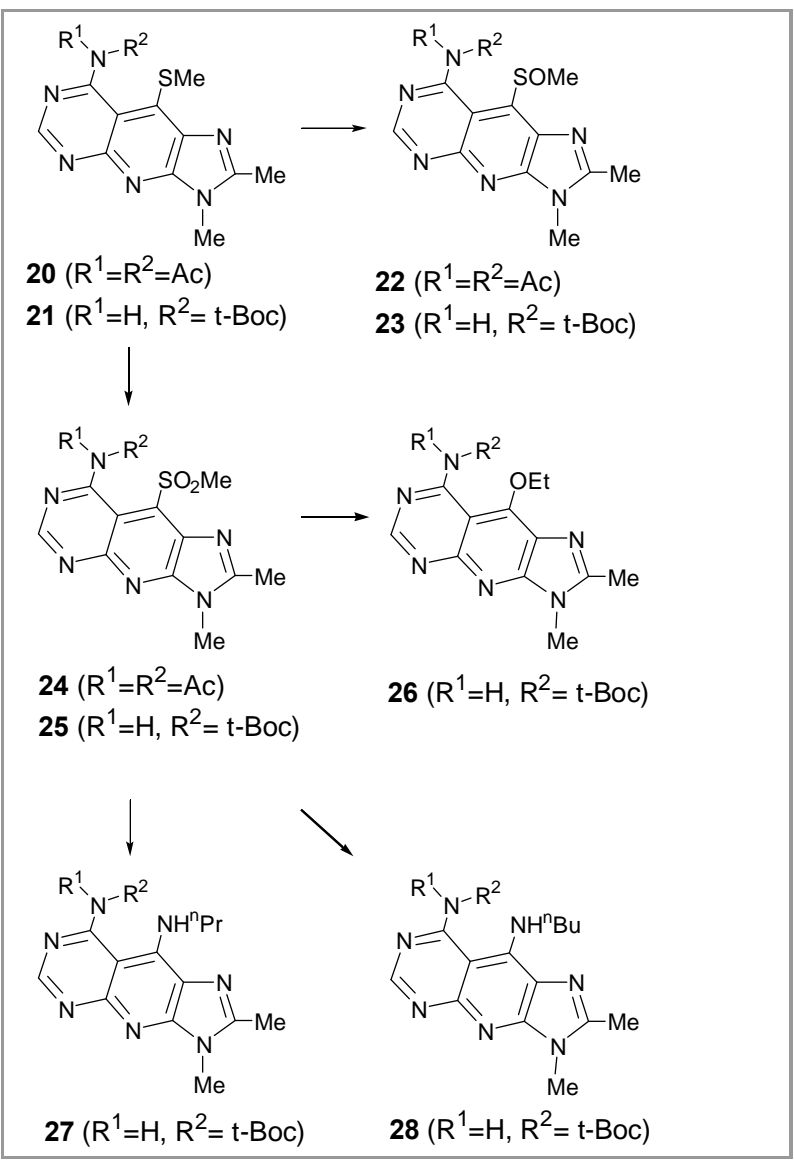

Scheme 2

The application of this methodology to the synthesis of 2ôdeoxyribonucleotide analogues requires the reaction of 2-(bis-methylsulfanylmethylene) malononitrile 9 with 5-aminoimidazole 29 to give the Cadduct 30. We have therefore made a preliminary investigation: reaction of the amine $\mathbf{2 9}^{11}$ with electrophile 9 gave a single product which after chromatography was identified as the desired adduct 30 (mp $98-100{ }^{\circ} \mathrm{C}, 15 \%$ ), which was fully characterized. The yield was low and requires optimization but the regioselectivity of the reaction demonstrates that this is a potential route to 9 -substituted derivatives.

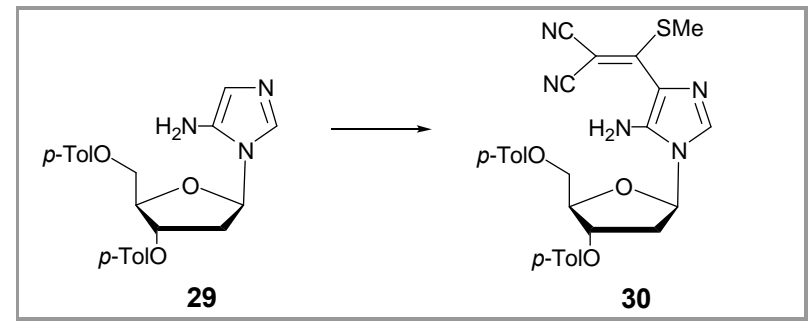

Scheme 3 
IR spectra were obtained using a Thermo-Nicolet Avatar 370 Fourier Transform Infra Red spectrometer. Mass spectra were obtained at the EPSRC National Mass Spectrometry Centre, University of Wales Swansea and were either low resolution electron impact (EI/LR), low resolution electrospray (ES/LR) or high resolution electrospray (ES/HR). Melting points were measured on a Stuart Scientific SMP3 melting point apparatus. NMR spectra were recorded on a Bruker Advance DPX300 NMR spectrometer in $\mathrm{CDCl}_{3}$ or $\mathrm{d}_{6}$-DMSO. Chemical shifts are quoted as ppm relative to TMS as internal standard. Solvents were dried as follows: THF was heated under reflux over, and then distilled from, sodium wire and benzophenone; DMF was dried by distillation, and then standing, over $4 \AA$ molecular sieves; pyridine was refluxed over $\mathrm{KOH}$ and allowed to stand over $4 \AA$ molecular sieves; 1,4-dioxane was refluxed over sodium and benzophenone, distilled off and stored over molecular sieves under a nitrogen atmosphere. TLC was carried out on aluminium backed $0.2 \mathrm{~mm}$ silica gel and visualised with $254 \mathrm{~nm}$ fluorescent indicator. Microanalyses were conducted through the Elemental Analysis Service at London Metropolitan University. AM1 calculations ${ }^{17}$ were performed using the MOPAC programme in CS Chem3D (CambridgeSoft Corporation). Calculated frontier orbital energies vary with configuration but variations in the values recorded in Table 1 do not change the conclusions.

2-(Bis-methylsulfanylmethylene)malononitrile $9^{16}$ and 2-(methylsulfanyl-phenylaminomethylene) malononitrile $11^{18}$ were prepared by literature methods.

\section{2-(Methlysulfanyl-propylaminomethylene)- malononitrile 10}

2-(Bis-methylsulfanylmethylene)malononitrile 9 (4.00 $\mathrm{g}, 23.5 \mathrm{mmol})$ was dissolved in ethanol $(25 \mathrm{~mL})$ with stirring. $n$-Propylamine $(1 \mathrm{~mL}, 11.8 \mathrm{mmol})$ was added dropwise over one hour and the mixture left to stir overnight. The solvent was then removed under reduced pressure and the solid product purified by column chromatography eluting with $\mathrm{CH}_{2} \mathrm{Cl}_{2}$ to remove the starting material followed by $5 \% \mathrm{MeOH}$ in $\mathrm{CH}_{2} \mathrm{Cl}_{2}$ to give the product 10 (1.66 g, 78\%); pale pink solid; mp 119-122 ${ }^{\circ} \mathrm{C}$.

IR (KBr): 632, 864, 920, 1153, 1248, 1290, 1302, 1364, 1419, 1442, 1467, 1500, 1548, 2187, 2206, $2877,2935,2964,3306 \mathrm{~cm}^{-1}$.

${ }^{1} \mathrm{H}$ NMR $\left(\mathrm{CDCl}_{3}\right): \delta=0.99(\mathrm{t}, J=7.0 \mathrm{~Hz}, 3 \mathrm{H}$, $\mathrm{NHCH}_{2} \mathrm{CH}_{2} \mathrm{CH}_{3}$ ), 1.67 (sextet, $J=7.0 \mathrm{~Hz}, 2 \mathrm{H}$, $\mathrm{NHCH}_{2} \mathrm{CH}_{2} \mathrm{CH}_{3}$ ), 2.68 (s, 3H, $\mathrm{SCH}_{3}$ ), 3.53 (q, $J=7.0$ $\mathrm{Hz}, 2 \mathrm{H}, \mathrm{NHCH}_{2} \mathrm{CH}_{2} \mathrm{CH}_{3}$ ), 6.31 (br s, $1 \mathrm{H}, \mathrm{NH}$ ).

${ }^{13} \mathrm{C} \mathrm{NMR}\left(\mathrm{CDCl}_{3}\right): \delta=11.00\left(\mathrm{NHCH}_{2} \mathrm{CH}_{2} \mathrm{CH}_{3}\right)$, $17.55 \quad\left(\mathrm{SCH}_{3}\right), \quad 23.31 \quad\left(\mathrm{NHCH}_{2} \mathrm{CH}_{2} \mathrm{CH}_{3}\right), \quad 48.33$ $\left(\mathrm{NHCH}_{2} \mathrm{CH}_{2} \mathrm{CH}_{3}\right), \quad 52.22\left(\mathrm{C}(\mathrm{CN})_{2}\right), \quad 115.35(\mathrm{CN})$, $115.64(C \mathrm{~N}), 174.69\left(C=\mathrm{C}(\mathrm{CN})_{2}\right)$.

MS (EI/LR): $m / z(\%)=181(38)\left[\mathrm{M}^{+}\right], 166(9), 152$ (18), 140 (13), 127 (15), 109 (12), 108 (19), 92 (58),

Template for SYNLETT and SYNTHESIS @ C Thieme Stuttgart · New York
79 (15), 74 (22), 68 (22), 61 (63), 48 (25), 47 (33), 45 (40), 43 (75), 41 (100).

Anal. Calcd for $\mathrm{C}_{8} \mathrm{H}_{11} \mathrm{~N}_{3} \mathrm{~S}$ (181.26): C, 53.01; H 6.12; N 23.18.

Found: C, 52.93; H, 6.17; N, 23.05.

2-[(5-Amino-1,2-dimethyl-1H-imidazol-4-yl)methylsulfanyl-methylene]malononitrile 13

1,2-Dimethyl-5-nitroimidazole $7\left(\mathrm{R}^{1}=\mathrm{R}^{2}=\mathrm{Me}\right)$ $(1.58 \mathrm{~g}, 11.2 \mathrm{mmol})$ and $5 \% \mathrm{Pd} / \mathrm{C}$ catalyst $(1.20 \mathrm{~g})$ were placed in a flask with dry THF $(125 \mathrm{~mL})$. The resulting mixture was hydrogenated at $\mathrm{rt}$ and atmospheric pressure, with vigorous stirring, over two hours. The reaction was monitored by TLC $\left(\mathrm{Et}_{2} \mathrm{O}\right)$ and showed the consumption of all starting material. The product mixture was filtered through dry celite, in an enclosed system under argon, into a flask containing 2-(bis-methylsulfanylmethylene) malononitrile 9 (9.64 $\mathrm{g}, 56.5 \mathrm{mmol})$. The reaction was stirred at $50{ }^{\circ} \mathrm{C}$, under argon, overnight giving an orange-brown solution. The solvent was removed under reduced pressure at rt and the residue treated with liquid nitrogen-chilled EtOAc $(100 \mathrm{~mL})$. The solution was then filtered to give a yellow-orange solid. The crude product 13 was used without further purification (yield over two stages $47 \%$ ).

An analytical sample was prepared by dissolving the solid in a minimum amount of $\mathrm{MeOH}$ followed by flash column chromatography (EtOAc as eluent). The relevant fractions were combined and the solvent removed by rotary evaporation giving a yellow solid that was recrystallised (EtOAc: $\mathrm{MeOH} \mathrm{5:3)} \mathrm{to} \mathrm{give} \mathrm{the}$ product $13(0.70 \mathrm{~g}, 27 \%)$; brilliant yellow crystals; $\mathrm{mp}$ 197-198 ${ }^{\circ} \mathrm{C}$.

IR (KBr): 1313, 1489, 1603, 2213, 2251, $3469 \mathrm{~cm}^{-1}$.

${ }^{1} \mathrm{H}$ NMR $\left(\mathrm{d}_{6}-\mathrm{DMSO}\right): \delta=2.23\left(\mathrm{~s}, 3 \mathrm{H}, \mathrm{S}-\mathrm{CH}_{3}\right), 2.49$ (s, 3H, C-CH $\left.H_{3}\right), 3.34$ (s, 3H, N-CH $\left.H_{3}\right), 7.05$ (br s, 2H, $\mathrm{N} \mathrm{H}_{2}$ ).

${ }^{13} \mathrm{C}$ NMR $\left(\mathrm{d}_{6}\right.$-DMSO): $\delta=13.22\left(\mathrm{C}-\mathrm{CH}_{3}\right), 17.81(\mathrm{~S}-$ $\left.\mathrm{CH}_{3}\right), 29.38\left(\mathrm{~N}-\mathrm{CH}_{3}\right), 60.64$ (methylene- $\left.\mathrm{C} 1\right), 113.70$ (imidazole- $C 5), 116.85(C \mathrm{~N}), 143.52$ (imidazole- $C 4)$, 147.64 (imidazole- $C 2$ ), 164.26 (methylene- $C 2$ ).

MS (EI/LR): $m / z(\%)=233(100)\left[\mathrm{M}^{+}\right], 218(8), 200$ (22), 187 (21), 161 (17), 123 (4), 104 (10), 84 (22), 56 (63), 49 (29), 42 (24).

Anal. Calcd for $\mathrm{C}_{10} \mathrm{H}_{11} \mathrm{~N}_{5} \mathrm{~S}$ (233.29): C, 51.48; H, $4.75 ; \mathrm{N}, 30.02 \%$.

Found: C, 51.53; H, 4.86; N, 29.84\%.

\section{5-Amino-2,3-dimethyl-7-methylsulfanyl-3H- imidazo[4,5-b]pyridine-6-carbonitrile 14}

2-[(5-Amino-1,2-dimethyl- $1 H$-imidazol-4-yl)methylsulfanyl-methylene]malononitrile $13(0.20 \mathrm{~g}$, $0.86 \mathrm{mmol})$ was placed in a flask with $\mathrm{MeOH}(50 \mathrm{~mL})$ and heated to $60{ }^{\circ} \mathrm{C}$. $\mathrm{NaOH}(0.18 \mathrm{~g})$ in $\mathrm{H}_{2} \mathrm{O}(2 \mathrm{~mL})$ was added to the reaction mixture. The reaction was followed by TLC (EtOAc: $\mathrm{MeOH} 10: 1$ ) and after 20 
min. showed one fluorescent spot (rf 0.67). The mixture was cooled to $\mathrm{rt}$ and the precipitate collected, recrystallised from DMF and identified as the product 14 (0.17 g, 86\%); off-white crystals; mp $300{ }^{\circ} \mathrm{C}$.

IR (KBr): 517, 639, 899, 1370, 1425, 1488, 1560, $1585,1648,2202,2931,3338,3412 \mathrm{~cm}^{-1}$.

${ }^{1} \mathrm{H}$ NMR $\left(\mathrm{d}_{6}\right.$-DMSO): $\delta=2.45\left(\mathrm{~s}, 3 \mathrm{H}, \mathrm{C}-\mathrm{CH}_{3}\right), 3.03$ (s, 3H, S-CH $\left.H_{3}\right), 3.54$ (s, 3H, N-CH $\left.H_{3}\right), 6.54$ (br s, $2 \mathrm{H}$, $\mathrm{N} H_{2}$ ).

${ }^{13} \mathrm{C}$ NMR (d 6 -DMSO): $\delta=14.38\left(\mathrm{C}-\mathrm{CH}_{3}\right), 16.74(\mathrm{~S}-$ $\left.\mathrm{CH}_{3}\right), 28.64\left(\mathrm{~N}-\mathrm{CH}_{3}\right), 83.63(\mathrm{C} 6), 117.20(\mathrm{CN})$, 126.01 (C1a), 144.52 (C3a), 149.82 (C2), 151.59 (C7), 157.94 (C5).

MS (EI/LR): $m / z(\%)=233(100)\left[\mathrm{M}^{+}\right], 232(24), 218$ (9), 206 (11), 200 (25), 188 (12), 187 (27), 186 (15), 145 (13), 123 (12), 117 (12), 109 (18), 105 (23), 104 (44), 95 (21), 91 (45), 82 (44), 77 (56), 69 (34), 67 (43).

Anal. Calcd for $\mathrm{C}_{10} \mathrm{H}_{11} \mathrm{~N}_{5} \mathrm{~S}$ (233.29): C, 51.48; H, 4.75; N, 30.02 .

Found: C, 51.33; H, 4.67; N, 29.90.

$\mathrm{N}$-(6-Cyano-2,3-dimethyl-7-methylsulfanyl-3Himidazo[4,5-b]pyridin-5-yl)-formimidic acid ethyl ester 15

5-Amino-2,3-dimethyl-7-methylsulfanyl-3Himidazo[4,5- $b$ ]pyridine-6-carbonitrile 14 (5.96 g, 25.5 mmol), triethyl orthoformate $(500 \mathrm{~mL})$ and paratoluenesulphonic acid monohydrate $(0.81 \mathrm{~g}, 4.3$ mmol) were placed in a flask fitted with a Claisen head and condenser. The mixture was heated to 150 ${ }^{\circ} \mathrm{C}(4 \mathrm{~h})$. Activated carbon was then added and the hot mixture filtered. The precipitate, which appeared on cooling, was collected and washed with a little $\mathrm{Et}_{2} \mathrm{O}$ giving the product 15 (6.43 g, 87\%); fine colourless crystals; mp $197-200{ }^{\circ} \mathrm{C}$.

IR (KBr): 1196, 1226, 1559, 1580, 1627, 2214, 2935 $\mathrm{cm}^{-1}$.

${ }^{1} \mathrm{H}$ NMR (d $\mathrm{d}_{6}$-DMSO): $\delta=1.36(\mathrm{t}, J=7.0 \mathrm{~Hz}, 3 \mathrm{H}$, $\mathrm{CH}_{3} \mathrm{CH}_{2}$ ), 2.56 (s, 3H, C-CH3), 3.11 (s, 3H, S-CH $\mathrm{CH}_{3}$, $3.69\left(\mathrm{~s}, 3 \mathrm{H}, \mathrm{N}-\mathrm{CH}_{3}\right), 4.37$ (q, $J=7.0 \mathrm{~Hz}, 2 \mathrm{H}$, $\mathrm{CH}_{3} \mathrm{CH}_{2}$ ), 8.56 (s, $1 \mathrm{H}, \mathrm{CH}-\mathrm{OEt}$ ).

${ }^{13} \mathrm{C}$ NMR $\left(\mathrm{d}_{6}\right.$-DMSO): $\delta=13.84\left(\mathrm{C}-\mathrm{CH}_{3}\right), 13.98$ $\left(\mathrm{CH}_{3} \mathrm{CH}_{2}\right), \quad 16.28\left(\mathrm{~S}-\mathrm{CH}_{3}\right), 28.35\left(\mathrm{~N}-\mathrm{CH}_{3}\right), 63.34$ $\left(\mathrm{CH}_{3} \mathrm{CH}_{2}\right), 94.81(\mathrm{C} 6), 115.78(\mathrm{CN}), 130.10$ (C1a), 144.19 (C3a), 147.75 (C2), 153.98 (C7), 156.10 $(\mathrm{EtO} C=\mathrm{N}), 160.45(C 5)$.

MS (EI): $m / z(\%)=289(21)\left[\mathrm{M}^{+}\right], 260(100), 244$ (46), 232 (15), 186 (12), 171 (10), 145 (12), 135 (12), 104 (19), 91 (15), 77 (13), 56 (57), 46 (12), 42 (18).

Anal. Calcd for $\mathrm{C}_{13} \mathrm{H}_{15} \mathrm{~N}_{5} \mathrm{OS}$ (289.36): C, 53.92; H, 5.22; N, 24.29.

Found: C, 53.75; H, 5.00; N, 24.07.
2,3-Dimethyl-9-methylsulfanyl-3H-1,3,4,5,7pentaaza-cyclopenta $[b]$ naphthalen-8-ylamine 16

$\mathrm{N}$-(6-Cyano-2,3-dimethyl-7-methylsulfanyl-3Himidazo[4,5-b]pyridin-5-yl)-formimidic acid ethyl ester 15 (3.03 g, $10.5 \mathrm{mmol})$ was added to EtOH (300 $\mathrm{mL})$ saturated with ammonia. The mixture was stirred at $\mathrm{rt}(1 \mathrm{~h})$ and then heated under reflux overnight. The mixture was cooled in a freezer and the precipitate collected and identified as the product 16 (2.31 g, $85 \%$ ); fine colourless crystals; mp 252-255 ${ }^{\circ} \mathrm{C}$. An analytical sample was recrystallised from isopropanol.

IR (KBr): 1346, 1401, 1429, 1455, 1500, 1521, 1551, $1576,1638,2360,2937,3075,3282,3434 \mathrm{~cm}^{-1}$.

${ }^{1} \mathrm{H}$ NMR (d $\mathrm{d}_{6}$-DMSO): $\delta=2.66\left(\mathrm{~s}, 3 \mathrm{H}, \mathrm{C}-\mathrm{CH}_{3}\right), 2.94$ (s, 3H, S-CH $\mathrm{H}_{3}$ ) 3.75 (s, 3H, N-CH $\left.H_{3}\right), 8.32$ (br s, $2 \mathrm{H}$, $\left.\mathrm{N} \mathrm{H}_{2}\right), 8.40$ (s, $\left.1 \mathrm{H}, \mathrm{CH}\right)$.

${ }^{13} \mathrm{C}$ NMR (d 6 -DMSO): $\delta=14.45\left(\mathrm{C}-\mathrm{CH}_{3}\right), 19.48(\mathrm{~S}-$ $\left.\mathrm{CH}_{3}\right), 28.27\left(\mathrm{~N}-\mathrm{CH}_{3}\right), 105.43(\mathrm{C} 8 \mathrm{a}), 133.47$ (C1a), 135.41 (C3a), 150.56 (C9), 155.78 (C2), 155.90 (C6), 157.88 (C4a), $163.70(C 8)$.

MS (EI): $m / z(\%)=260(88)\left[\mathrm{M}^{+}\right], 254(13), 245(21)$, 234 (12), 233 (53), 227 (75), 218 (16), 214 (22), 212 (44), 210 (68), 204 (71), 197 (100), 187 (85), 179 (59), 169 (62).

Anal. Calcd for $\mathrm{C}_{11} \mathrm{H}_{12} \mathrm{~N}_{6} \mathrm{~S}$ (260.32): C, 50.75; H, 4.65; N, 32.28.

Found: C, 50.60; H, 4.44; N, 32.28.

\section{2,3-Dimethyl-9-methylsulfanyl-3,7-dihydro- $1,3,4,5,7-$ pentaaza-cyclopenta $[b]$ naphthalen-8-one 17}

2,3-Dimethyl-9-methylsulfanyl-3H-1,3,4,5,7pentaaza-cyclopenta[$[b]$ naphthalen-8-ylamine 16 (1.00 $\mathrm{g}, 3.8 \mathrm{mmol})$ in $2 \mathrm{M} \mathrm{HCl}(100 \mathrm{~mL})$ was heated under reflux $(2 \mathrm{~h})$. The solution was cooled in an ice bath and basified with aqueous ammonia. The resulting precipitate was collected, washed with $\mathrm{H}_{2} \mathrm{O}$, EtOH and finally $\mathrm{Et}_{2} \mathrm{O}$ giving the product $17(0.74 \mathrm{~g}, 74 \%)$; colourless solid; $\mathrm{mp}>350{ }^{\circ} \mathrm{C}$.

IR (KBr): 956, 1242, 1314, 1324, 1351, 1373, 1423, $1472,1548,1575,1615,1668,2629,2910,3046 \mathrm{~cm}^{-1}$.

${ }^{1} \mathrm{H}$ NMR (TFA): $\delta=3.12\left(\mathrm{~s}, 3 \mathrm{H}, \mathrm{C}-\mathrm{CH}_{3}\right), 3.15(\mathrm{~s}, 3 \mathrm{H}$, $\left.\mathrm{S}-\mathrm{CH}_{3}\right), 4.17$ (s, 3H, N-CH3), $9.56(\mathrm{~s}, 1 \mathrm{H}, \mathrm{CH})$.

${ }^{13} \mathrm{C}$ NMR (TFA): $\delta=14.74\left(\mathrm{C}-\mathrm{CH}_{3}\right), 20.18\left(\mathrm{~S}-\mathrm{CH}_{3}\right)$, $33.08\left(\mathrm{~N}-\mathrm{CH}_{3}\right), 126.42$ (C8a), 149.03 (C1a), 151.31 (C3a), 154.42 (C9), 155.07 (C2), 161.66 (C6), 161.99 (C4a), 166.41 (C8).

MS (EI): $m / z(\%)=261(31)\left[\mathrm{M}^{+}\right], 228$ (15), 187 (9), 105 (10), 91 (17), 77 (9), 56 (12), 44 (100).

Anal. Calcd for $\mathrm{C}_{11} \mathrm{H}_{11} \mathrm{~N}_{5} \mathrm{SO}$ (261.30): C, 50.56; H, 4.24; N, 26.80.

Found: C, 50.54; H, 4.06; N, 26.65. 
$\mathrm{N}$-(6-Cyano-2,3-dimethyl-7-methylsulfanyl-3Himidazo[4,5-b]pyridin-5-yl)- $N$ '-propylformamidine 18

$\mathrm{N}$-(6-cyano-2,3-dimethyl-7-methylsulfanyl-3Himidazo[4,5-b]pyridin-5-yl)-formimidic acid ethyl ester $15(0.50 \mathrm{~g}, 1.7 \mathrm{mmol})$ and $n$-propylamine $(0.70$ $\mathrm{mL}, 8.5 \mathrm{mmol})$ in $\mathrm{EtOH}(25 \mathrm{~mL})$ were allowed to stand at $\mathrm{rt}(18 \mathrm{~h})$. The solvent and excess amine were removed under reduced pressure. The solid residue was recrystallised (EtOAc and petroleum ether) and identified as the product $18(0.31 \mathrm{~g}, 58 \%)$; off-white crystals; mp $147-149{ }^{\circ} \mathrm{C}$.

IR (KBr): 938, 959, 1151, 1195, 1250, 1349, 1373, $1423,1475,1574,1615,2212,2873,2929,2958$, $3243 \mathrm{~cm}^{-1}$.

${ }^{1} \mathrm{H}$ NMR $\left(\mathrm{d}_{6}\right.$-DMSO): $\delta=0.97(\mathrm{t}, J=7.5 \mathrm{~Hz}, 3 \mathrm{H}$, $\left.\mathrm{NHCH}_{2} \mathrm{CH}_{2} \mathrm{CH}_{3}\right), 1.64\left(\mathrm{~m}, 2 \mathrm{H}, \mathrm{NHCH}_{2} \mathrm{CH}_{2} \mathrm{CH}_{3}\right)$, $3.05\left(\mathrm{~s}, 3 \mathrm{H}, \mathrm{C}-\mathrm{CH}_{3}\right), 3.34$ (s, $\left.3 \mathrm{H}, \mathrm{S}-\mathrm{CH}_{3}\right), 3.38(\mathrm{q}, J=$ $\left.6.5 \mathrm{~Hz}, 2 \mathrm{H}, \mathrm{NHCH}_{2} \mathrm{CH}_{2} \mathrm{CH}_{3}\right), 3.62\left(\mathrm{~s}, 3 \mathrm{H}, \mathrm{N}-\mathrm{CH}_{3}\right)$, $8.09(\mathrm{~d}, J=4.5 \mathrm{~Hz}, 1 \mathrm{H}, \mathrm{CH}), 8.52(\mathrm{~d}, J=4.5 \mathrm{~Hz}, 1 \mathrm{H}$, $\mathrm{N} H$ ).

${ }^{13} \mathrm{C}$ NMR $\left(\mathrm{d}_{6}\right.$-DMSO): $\delta=11.44\left(\mathrm{NHCH}_{2} \mathrm{CH}_{2} \mathrm{CH}_{3}\right)$, $13.89 \quad\left(\mathrm{C}-\mathrm{CH}_{3}\right), \quad 16.17 \quad\left(\mathrm{~S}-\mathrm{CH}_{3}\right), \quad 21.49$ $\left(\mathrm{NHCH}_{2} \mathrm{CH}_{2} \mathrm{CH}_{3}\right), \quad 28.07 \quad\left(\mathrm{~N}-\mathrm{CH}_{3}\right), \quad 42.16$ $\left(\mathrm{NHCH}_{2} \mathrm{CH}_{2} \mathrm{CH}_{3}\right), 94.16(\mathrm{C6}), 116.89(\mathrm{CN}), 128.27$ (C1a), 143.56 (C3a), 148.16 (C2), 152.30 (C7), $153.21(\mathrm{~N}=C-\mathrm{N}), 160.11(C 5)$.

MS (EI): $m / z(\%)=302(26)\left[\mathrm{M}^{+}\right], 287$ (10), 269 (12), 244 (9), 200 (13), 187 (22), 56 (14).

Anal. Calcd for $\mathrm{C}_{14} \mathrm{H}_{18} \mathrm{~N}_{6} \mathrm{~S}$ (302.4): C, 55.61; H, 6.00; N, 27.79.

Found: C, 55.64; H, 5.79; N, 27.61.

\section{$\mathrm{N}$-Acetyl- $\mathrm{N}$-(2,3-dimethyl-9-methylsulfanyl-3H- 1,3,4,5,7-pentaaza-cyclopenta $[b]$ naphthalen-8-yl)- acetamide 20}

To a suspension of 2,3-dimethyl-9-methylsulfanyl-3 $\mathrm{H}$ 1,3,4,5,7-pentaaza-cyclopenta[b]naphthalen-8-

ylamine $16(2.00 \mathrm{~g}, 7.7 \mathrm{mmol})$ in pyridine $(27 \mathrm{~mL})$ was added $\mathrm{Ac}_{2} \mathrm{O}(20 \mathrm{~mL})$. The mixture was heated under reflux $(2 \mathrm{~h})$ and then poured onto ice water (50 $\mathrm{mL})$. The product was extracted into $\mathrm{CH}_{2} \mathrm{Cl}_{2}(2 \mathrm{x}$ $50 \mathrm{~mL})$, dried $\left(\mathrm{MgSO}_{4}\right)$ and the solvent reduced by half $(\sim 20 \mathrm{~mL}) . \quad \mathrm{Et}_{2} \mathrm{O}$ was added and the solution left to cool overnight. The precipitate was collected, dried under high vacuum at $100{ }^{\circ} \mathrm{C}$ and identified as the product 20 (2.01 g, 76\% yield); fine yellow crystals; mp $178-183{ }^{\circ} \mathrm{C}$.

IR (KBr): 1163, 1219, 1296, 1353, 1404, 1479, 1539, 1574, 1608, 1731, 2341, 2359, 2930, $3007 \mathrm{~cm}^{-1}$.

${ }^{1} \mathrm{H} \mathrm{NMR}\left(\mathrm{CDCl}_{3}\right): \delta=2.30\left(\mathrm{~s}, 6 \mathrm{H}, \mathrm{NC}(\mathrm{O}) \mathrm{CH}_{3}\right), 2.66$ $\left(\mathrm{s}, 3 \mathrm{H}, \mathrm{C}-\mathrm{CH}_{3}\right), 3.17\left(\mathrm{~s}, 3 \mathrm{H}, \mathrm{S}-\mathrm{CH}_{3}\right), 3.86(\mathrm{~s}, 3 \mathrm{H}, \mathrm{N}-$ $\left.\mathrm{CH}_{3}\right), 9.29(\mathrm{~s}, 1 \mathrm{H}, \mathrm{CH})$.

${ }^{13} \mathrm{C}$ NMR $\left(\mathrm{CDCl}_{3}\right): \delta=15.01\left(\mathrm{C}-\mathrm{CH}_{3}\right), 20.11(\mathrm{~S}-$ $\left.\mathrm{CH}_{3}\right), 27.10\left(\mathrm{NC}(\mathrm{O}) \mathrm{CH}_{3}\right) 28.93\left(\mathrm{~N}-\mathrm{CH}_{3}\right), 115.15$
(C8a), 136.06 (C1a), 138.28 (C3a), 152.94 (C9), 155.70 (C2), 157.79 (C6), 157.85 (C4a), 160.60 (C8), $171.67\left(\mathrm{NC}(\mathrm{O}) \mathrm{CH}_{3}\right)$.

MS (EI): $m / z(\%)=344$ (3) $\left[\mathrm{M}^{+}\right], 301$ (3), 269 (12), 255 (19), 245 (12), 227 (3), 171 (6), 135 (2), 94 (6), 82 (3), 56 (12), 43 (100).

Anal. Calcd for $\mathrm{C}_{15} \mathrm{H}_{16} \mathrm{~N}_{6} \mathrm{O}_{2} \mathrm{~S}$ (344.39): $\mathrm{C}, 52.31 ; \mathrm{H}$, 4.68; N, 24.40.

Found: C, 52.43; H, 4.36; N, 24.19.

(2,3-Dimethyl-9-methanesulfanyl-3H-1,3,4,5,7pentaaza-cyclopenta $[b]$ naphthalen-8-yl)-carbamic acid tert-butyl ester 21

2,3-Dimethyl-9-methylsulfanyl-3H-1,3,4,5,7pentaaza-cyclopenta[ $b]$ naphthalen-8-ylamine 16 (1.00 $\mathrm{g}, 3.8 \mathrm{mmol})$ was suspended in dry 1,4-dioxane (100 $\mathrm{mL}$ ) and heated to $120{ }^{\circ} \mathrm{C}$. When all the starting material had dissolved, di-tert-butyl dicarbonate $(4.19 \mathrm{~g}$, $19.2 \mathrm{mmol}$ ) was added and the solution stirred $(5 \mathrm{~h})$ after which TLC $(\mathrm{MeOH})$ showed that all starting material had reacted. The solvent was removed by evaporation and the resulting solid treated with $\mathrm{Et}_{2} \mathrm{O}$ $(50 \mathrm{~mL})$. The solid was then collected, washed with further $\mathrm{Et}_{2} \mathrm{O}(2 \times 25 \mathrm{~mL})$ and identified as the product 21 ( $1.15 \mathrm{~g}, 83 \%$ yield); yellow solid; $\mathrm{mp} 167{ }^{\circ} \mathrm{C}$ (decomp.).

IR (KBr): 1060, 1152, 1248, 1305, 1353, 1372, 1426, $1505,1580,1697,1758,2923,2976,3422 \mathrm{~cm}^{-1}$.

${ }^{1} \mathrm{H}$ NMR $\left(\mathrm{CDCl}_{3}\right): \delta=1.61\left(\mathrm{~s}, 9 \mathrm{H}, \mathrm{C}\left(\mathrm{CH}_{3}\right)_{3}\right), 2.77(\mathrm{~s}$, $\left.3 \mathrm{H}, \mathrm{C}-\mathrm{CH}_{3}\right), 2.87\left(\mathrm{~s}, 3 \mathrm{H}, \mathrm{S}-\mathrm{CH}_{3}\right), 3.91(\mathrm{~s}, 3 \mathrm{H}, \mathrm{N}-$ $\left.\mathrm{CH}_{3}\right), 9.10(\mathrm{~s}, 1 \mathrm{H}, \mathrm{CH}), 11.49$ (br s, $1 \mathrm{H}, \mathrm{N} H$ ).

${ }^{13} \mathrm{C}$ NMR $\left(\mathrm{CDCl}_{3}\right): \delta=15.08\left(\mathrm{C}-\mathrm{CH}_{3}\right), 20.74(\mathrm{~S}-$ $\left.\mathrm{CH}_{3}\right), \quad 28.25 \quad\left(\mathrm{O}-\left(\mathrm{CH}_{3}\right)_{3}\right), \quad 28.97 \quad\left(\mathrm{~N}-\mathrm{CH}_{3}\right), \quad 82.21$ ((Me) $\left.)_{3}-C-\mathrm{O}\right), 108.08$ (C8a), 131.27 (C1a), 138.25 (C3a), 149.79 (C9), 151.74 (HN-COO), 156.09 (C2), 156.46 (C6), 158.64 (C4a), 159.72 (C8).

Anal. Calcd for $\mathrm{C}_{16} \mathrm{H}_{20} \mathrm{~N}_{6} \mathrm{O}_{2} \mathrm{~S}$ (360.44): C, 53.32; H, 5.59; N, 23.32.

Found: C, 53.03; H, 5.43; N, 23.57.

$\mathrm{N}$-Acetyl- $\mathrm{N}$-(9-methanesulfinyl-2,3-dimethyl-3H1,3,4,5,7-pentaaza-cyclopenta[b]naphthalen-8-yl)acetamide 22

$\mathrm{N}$-Acetyl- $\mathrm{N}$-(2,3-dimethyl-9-methylsulfanyl-3H1,3,4,5,7-pentaaza-cyclopenta[ $b]$ naphthalen-8-yl)acetamide 20 (194 mg, $0.56 \mathrm{mmol}$ ) was dissolved in $\mathrm{CH}_{2} \mathrm{Cl}_{2} \quad(20 \mathrm{~mL})$ and cooled to $0{ }^{\circ} \mathrm{C}$. 3Chloroperoxybenzoic acid $(50-55 \%)(194 \mathrm{mg}, 0.56$ $\mathrm{mmol})$ was added and the mixture stirred $(1.5 \mathrm{~h})$. The organic layer was then washed with sat. aq $\mathrm{NaHCO}_{3}$ ( $2 \times 20 \mathrm{~mL})$ and $\mathrm{H}_{2} \mathrm{O}(2 \times 20 \mathrm{~mL})$. The organic layer was then dried $\left(\mathrm{MgSO}_{4}\right)$ and the solvent removed under reduced pressure. The crude material was dissolved in EtOAc and the solvent reduced until crystallisation began. After cooling, the solid was collected 
and identified as the product 22 (22 $\mathrm{mg}, 11 \%$ yield); yellow crystals; mp 191-192 ${ }^{\circ} \mathrm{C}$.

IR (KBr): 568, 599, 640, 685, 926, 867, 1006, 1040, 1067, 1157, 1230, 1301, 1369, 1423, 1475, 1514, $1577,1616,1698,1720,2922,3006 \mathrm{~cm}^{-1}$.

${ }^{1} \mathrm{H}$ NMR $\left(\mathrm{CDCl}_{3}\right): \delta=2.12\left(\mathrm{~s}, 3 \mathrm{H}, \mathrm{CO}-\mathrm{CH}_{3}\right), 2.65 \mathrm{~s}$, $\left(3 \mathrm{H}, \mathrm{CO}-\mathrm{CH}_{3}\right), 2.88$ (s, 3H, C-CH $\left.\mathrm{CH}_{3}\right), 3.45$ (s, 3H, SO$\left.\mathrm{CH}_{3}\right), 4.01\left(\mathrm{~s}, 3 \mathrm{H}, \mathrm{N}-\mathrm{CH}_{3}\right), 9.46(\mathrm{~s}, 1 \mathrm{H}, \mathrm{CH})$.

${ }^{13} \mathrm{C}$ NMR $\left(\mathrm{CDCl}_{3}\right): \delta=15.97\left(\mathrm{C}-\mathrm{CH}_{3}\right), 26.76$ $\left(\mathrm{NC}(\mathrm{O}) \mathrm{CH}_{3}\right), 28.15\left(\mathrm{NC}(\mathrm{O}) \mathrm{CH}_{3}\right), 29.69\left(\mathrm{~N}-\mathrm{CH}_{3}\right)$, $40.86\left(\mathrm{SO}-\mathrm{CH}_{3}\right), 113.77$ (C8a), 136.33 (C3a), 138.37 (C1a), 156.00 (C9), 156.36 (C2), 156.82 (C6), 160.42 $(C 4 \mathrm{a}), 164.18$ (C8), $170.72\left(\mathrm{NC}(\mathrm{O}) \mathrm{CH}_{3}\right), 174.51$ $\left(\mathrm{NC}(\mathrm{O}) \mathrm{CH}_{3}\right)$.

MS (EI): $m / z(\%)=361(3)\left[\mathrm{M}+\mathrm{H}^{+}\right], 345(9), 303(6)$, 277 (4), 255 (85), 246 (6), 200 (6), 120 (26), 119 (54), 111 (8), 97 (12), 77 (100).

Anal. Calcd for $\mathrm{C}_{15} \mathrm{H}_{16} \mathrm{~N}_{6} \mathrm{O}_{3} \mathrm{~S}$ (360.39): C, 49.99; H, 4.47; N, 23.32.

Found: C, 49.73; H, 4.52; N, 23.06.

(9-Methanesulfinyl-2,3-dimethyl-3H-1,3,4,5,7pentaaza-cyclopenta $[b]$ naphthalen-8-yl)-carbamic acid tert-butyl ester 23

Using the procedure described for compound 22, compound 23 was obtained from (2,3-dimethyl-9methanesulfanyl-3H-1,3,4,5,7-pentaaza-

cyclopenta $[b]$ naphthalen-8-yl)-carbamic acid tertbutyl ester 21 (1.00 g, $2.8 \mathrm{mmol})$; yield: $0.66 \mathrm{~g}, 63 \%$; pale yellow, crystalline solid; mp $172{ }^{\circ} \mathrm{C}$ (decomp.).

IR (KBr): 749, 812, 839, 879, 927, 958, 1022, 1074, $1146,1225,1252,1307,1373,1429,1493,1527$, 1588, 1752, 2728, 2927, 2978, $3494 \mathrm{~cm}^{-1}$.

${ }^{1} \mathrm{H} \mathrm{NMR}\left(\mathrm{CDCl}_{3}\right): \delta=1.59\left(\mathrm{~s}, 9 \mathrm{H}, \mathrm{C}\left(\mathrm{CH}_{3}\right)_{3}\right), 2.79(\mathrm{~s}$, $\left.3 \mathrm{H}, \mathrm{C}-\mathrm{CH}_{3}\right), 3.12$ (s, 3H, SO-CH$H_{3}, 3.95$ (s, 3H, N$\mathrm{CH}_{3}$ ), 9.22 (s, $\left.1 \mathrm{H}, \mathrm{CH}\right), 12.28$ (br s, $1 \mathrm{H}, \mathrm{NH}$ ).

${ }^{13} \mathrm{C}$ NMR $\left(\mathrm{CDCl}_{3}\right): \delta=15.56\left(\mathrm{C}-\mathrm{CH}_{3}\right), 28.66(\mathrm{O}-$ $\left.\mathrm{C}\left(\mathrm{CH}_{3}\right)_{3}\right), 29.57\left(\mathrm{~N}-\mathrm{CH}_{3}\right), \quad 39.84\left(\mathrm{SO}-\mathrm{CH}_{3}\right), \quad 82.18$ ((Me) $\left.{ }_{3}-C-\mathrm{O}\right), 107.99$ (C8a), 134.44 (C3a), 136.30 (C1a), 151.54 (C2), 153.33 (HN-COO), 157.30 (C9), 158.27 (C6), 158.81 (C4a), 162.37 (C8).

\section{$\mathrm{N}$-Acetyl- $\mathrm{N}$-(9-methanesulfonyl-2,3-dimethyl-3H- $1,3,4,5,7-$ pentaaza-cyclopenta $[b]$ naphthalen-8-yl)- acetamide 24}

$\mathrm{N}$-Acetyl- $\mathrm{N}$-(2,3-dimethyl-9-methylsulfanyl-3H1,3,4,5,7-pentaaza-cyclopenta[b]naphthalen-8-yl)acetamide 20 (2.00 g, $5.8 \mathrm{mmol})$ was dissolved in $\mathrm{CH}_{2} \mathrm{Cl}_{2} \quad(50 \mathrm{~mL})$ and cooled to $0{ }^{\circ} \mathrm{C}$. 3Chloroperoxybenzoic acid (50-55\%) (4.00 g, 11.6 mmol) was added and the mixture stirred $(4 \mathrm{~h})$. The organic layer was diluted with a further portion of $\mathrm{CH}_{2} \mathrm{Cl}_{2}(50 \mathrm{~mL})$, washed with $\mathrm{H}_{2} \mathrm{O}(2 \times 100 \mathrm{~mL})$ and dried $\left(\mathrm{MgSO}_{4}\right)$. After the solvent had been removed under reduced pressure, the crude solid was recrystallised from EtOAc and identified as the product $\mathbf{2 4}$ (1.51 g, 67\%); orange crystals; mp $185-186^{\circ} \mathrm{C}$.

IR (KBr): 537, 579, 640, 755, 933, 1009, 1038, 1122, $1152,1211,1236,1259,1310,1353,1366,1404$, $1426,1485,1517,1561,1577,1623,1706,1733$, $2924,3016 \mathrm{~cm}^{-1}$

${ }^{1} \mathrm{H}$ NMR $\left(\mathrm{CDCl}_{3}\right): \delta=2.48\left(\mathrm{~s}, 6 \mathrm{H}, \mathrm{CO}-\mathrm{CH}_{3}\right), 2.81(\mathrm{~s}$, $\left.3 \mathrm{H}, \mathrm{C}-\mathrm{CH}_{3}\right), 3.83$ (s, 3H, $\left.\mathrm{SO}_{2}-\mathrm{CH}_{3}\right), 3.98$ (s, 3H, N$\left.\mathrm{CH}_{3}\right), 9.42$ (s, $\left.1 \mathrm{H}, \mathrm{CH}\right)$.

${ }^{13} \mathrm{C} \quad \mathrm{NMR}\left(\mathrm{CDCl}_{3}\right): \delta=15.44\left(\mathrm{C}-\mathrm{CH}_{3}\right), 27.89$ $\left(\mathrm{C}(\mathrm{O}) \mathrm{CH}_{3}\right), 29.36\left(\mathrm{~N}-\mathrm{CH}_{3}\right), 48.90\left(\mathrm{~S}-\mathrm{CH}_{3}\right), 112.17$ (C8a), 132.79 (C3a), 132.83 (C1a), 143.21 (C2), 155.55 (C9), 155.86 (C6), 160.25 (C4a), 163.78 (C8), $173.18\left(\boldsymbol{C}(\mathrm{O}) \mathrm{CH}_{3}\right)$.

MS (EI): m/z (\%) = $377(2 \%)\left[\mathrm{M}+\mathrm{H}^{+}\right], 299(4), 255$ (35), 215 (4), 200 (7), 177 (4), 163 (4), 140 (4), 119 (9), 111 (18), 97 (24), 77 (100), 60 (12).

Anal. Calcd for $\mathrm{C}_{15} \mathrm{H}_{16} \mathrm{~N}_{6} \mathrm{O}_{4} \mathrm{~S}$ (376.39): C, 49.87; $\mathrm{H}$, 4.28; N, 22.33.

Found: C, 47.69; H, 4.05; N, 22.11.

(9-Methanesulfonyl-2,3-dimethyl-3H-1,3,4,5,7pentaaza-cyclopenta[b]naphthalen-8-yl)carbamic acid tert-butyl ester 25

Using the procedure described for compound 24, compound $\mathbf{2 5}$ was obtained from (2,3-dimethyl-9methanesulfanyl-3H-1,3,4,5,7-pentaaza-

cyclopenta $[b]$ naphthalen-8-yl)-carbamic acid tertbutyl ester 21 (2.00 g, $5.5 \mathrm{mmol}$ ); yield: $1.42 \mathrm{~g}, 65 \%$; dark gold, crystalline solid; mp $100{ }^{\circ} \mathrm{C}$ (decomp.).

IR (KBr): 774, 840, 1145, 1218, 1245, 1297, 1353 , $1375,1414,1509,1578,1752,2925,2976,3422 \mathrm{~cm}^{-1}$. ${ }^{1} \mathrm{H} \mathrm{NMR}\left(\mathrm{CDCl}_{3}\right): \delta=1.59\left(\mathrm{~s}, 9 \mathrm{H}, \mathrm{C}\left(\mathrm{CH}_{3}\right)_{3}\right), 2.79$ (s, $\left.3 \mathrm{H}, \mathrm{C}-\mathrm{CH}_{3}\right), 3.92\left(\mathrm{~s}, 3 \mathrm{H}, \mathrm{SO}_{2}-\mathrm{CH}_{3}\right), 3.95(\mathrm{~s}, 3 \mathrm{H}, \mathrm{N}-$ $\mathrm{CH}_{3}$ ), 9.16 (s, 1H, CH), 10.03 (br s, $1 \mathrm{H}, \mathrm{N} H$ ).

${ }^{13} \mathrm{C} \mathrm{NMR}\left(\mathrm{CDCl}_{3}\right): \delta=15.69\left(\mathrm{C}-\mathrm{CH}_{3}\right), 28.53(\mathrm{O}-$ $\left.\mathrm{C}\left(\mathrm{CH}_{3}\right)_{3}\right), \quad 29.56\left(\mathrm{~N}-\mathrm{CH}_{3}\right), \quad 48.26\left(\mathrm{SO}_{2}-\mathrm{CH}_{3}\right), \quad 82.44$ ((Me) $\left.)_{3}-C-\mathrm{O}\right), 104.32$ (C8a), 132.17 (C3a), 133.95 (C1a), 150.91 (HN-COO), 154.93 (C2), 155.97 (C9), 156.83 (C6), 157.16 (C4a), 163.16 (C8).

(9-Ethoxy-2,3-dimethyl-3H-1,3,4,5,7-pentaazacyclopenta[b]naphthalen-8-yl)-carbamic acid tertbutyl ester 26

(9-Methanesulfonyl-2,3-dimethyl-3H-1,3,4,5,7pentaaza-cyclopenta $[b]$ naphthalen-8-yl)-carbamic acid tert-butyl ester 25 (0.50 g, $1.3 \mathrm{mmol})$ was dissolved in EtOH $(50 \mathrm{~mL})$ with stirring. Sodium metal $(0.10 \mathrm{~g}$, $2.5 \mathrm{mmol}$ ) was then added and the mixture stirred for 30 min. at which point TLC (EtOAc: $\mathrm{MeOH} \mathrm{5:1)}$ showed the disappearance of the starting material and a new slower running spot (rf: 0.38). The EtOH was removed under reduced pressure and the residue redissolved in $\mathrm{CH}_{2} \mathrm{Cl}_{2}(50 \mathrm{~mL})$. The organic layer was 
then washed with $\mathrm{H}_{2} \mathrm{O}(2 \times 50 \mathrm{~mL})$, dried $\left(\mathrm{MgSO}_{4}\right)$ and the solvent removed.The solid product was then purified by column chromatography (3:1 EtOAc:MeOH) and the relevant fractions combined to give compound 26 (0.11 g, 24\%); colourless solid; mp: $204{ }^{\circ} \mathrm{C}$ (decomp.).

IR (KBr): 815, 949, 1016, 1060, 1109, 1143, 1241, 1356, 1369, 1380, 1466, 1507, 1555, 1597, 1624, $1663,1751,2973,3342 \mathrm{~cm}^{-1}$.

${ }^{1} \mathrm{H} \mathrm{NMR}\left(\mathrm{CDCl}_{3}\right): \delta=1.58\left(\mathrm{~s}, 9 \mathrm{H}, \mathrm{C}\left(\mathrm{CH}_{3}\right)_{3}\right), 1.67(\mathrm{t}$, $\left.J=7.0 \mathrm{~Hz}, 3 \mathrm{H}, \mathrm{OCH}_{2} \mathrm{CH}_{3}\right) 2.65\left(\mathrm{~s}, 3 \mathrm{H}, \mathrm{C}-\mathrm{CH}_{3}\right), 3.86$ (s, 3H, N-CH $), 5.34$ (q, $J=7.0 \mathrm{~Hz}, 2 \mathrm{H}, \mathrm{OCH}_{2} \mathrm{CH}_{3}$ ), 9.01 (s, 1H, CH), 10.40 (br s, $1 \mathrm{H}, \mathrm{N} H$ ).

${ }^{13} \mathrm{C} \mathrm{NMR}\left(\mathrm{CDCl}_{3}\right): \delta=15.02\left(\mathrm{O}-\mathrm{CH}_{2} \mathrm{CH}_{3}\right), 15.75(\mathrm{C}-$ $\left.\mathrm{CH}_{3}\right), 28.54\left(\mathrm{O}-\mathrm{C}\left(\mathrm{CH}_{3}\right)_{3}\right), 29.26\left(\mathrm{~N}-\mathrm{CH}_{3}\right), 72.23(\mathrm{O}-$ $\left.\mathrm{CH}_{2} \mathrm{CH}_{3}\right), 82.10\left(\left(\mathrm{CH}_{3}\right)_{3}-\mathrm{C}-\mathrm{O}\right), 100.20(\mathrm{C} 8 \mathrm{a}), 122.51$ (C1a), 149.85 (C3a), 152.39 (HN-COO), 155.45 (C2), 155.63 (C6), 157.21 (C4a), 157.42 (C8), 158.92 (C9).

MS (EI): $m / z(\%)=359(14)\left[\mathrm{M}+\mathrm{H}^{+}\right], 259$ (100), 244 (24), 222 (67), 204 (24), 192 (22), 176 (22), 150 (30), 148 (42), 135 (53), 122 (71), 100 (64), 98 (76), 84 (76), 72 (86).

HRMS(ES): $m / z$ calcd for $\mathrm{C}_{17} \mathrm{H}_{22} \mathrm{~N}_{6} \mathrm{O}_{3} \quad\left[\mathrm{M}+\mathrm{H}^{+}\right]$: 359.1826; found: 359.1825 .

\section{(2,3-Dimethyl-9-propylamino-3H-1,3,4,5,7-} pentaaza-cyclopenta $[b]$ naphthalen-8-yl) carbamic acid tert-butyl ester 27

(9-Methanesulfonyl-2,3-dimethyl-3H-1,3,4,5,7pentaaza-cyclopenta $[b]$ naphthalen-8-yl)-carbamic acid tert-butyl ester 25 (0.86 g, $2.2 \mathrm{mmol})$ was dissolved in $\mathrm{CH}_{2} \mathrm{Cl}_{2}$ (75 mL). To this solution was added $n$ propylamine $(0.18 \mathrm{~mL}, 2.2 \mathrm{mmol})$ and stirring was maintained at $\mathrm{rt}(2 \mathrm{~h})$. The solvent was removed under reduced pressure and the resulting solid dried under high vacuum at $70{ }^{\circ} \mathrm{C}(12 \mathrm{~h})$ to give the product $\mathbf{2 0}$ (0.80 g, 98\%); dark yellow solid; mp $218{ }^{\circ} \mathrm{C}$ (decomp.).

IR (KBr): 1003, 1071, 1114, 1157, 1218, 1253, 1384, 1439, 1528, 1586, 1652, 1700, 2862, 2965, $3408 \mathrm{~cm}^{-1}$.

${ }^{1} \mathrm{H} \mathrm{NMR}\left(\mathrm{CDCl}_{3}\right): \delta=1.15(\mathrm{t}, J=7.0 \mathrm{~Hz}, 3 \mathrm{H}$, $\left.\mathrm{NHCH}_{2} \mathrm{CH}_{2} \mathrm{CH}_{3}\right), 1.53$ (s, $\left.\mathrm{C}\left(\mathrm{CH}_{3}\right)_{3}\right), 1.77(\mathrm{~m}, 2 \mathrm{H}$, $\left.\mathrm{NHCH}_{2} \mathrm{CH}_{2} \mathrm{CH}_{3}\right), 2.55$ (s, 3H, C-CH $), 3.74$ (s, 3H, $\left.\mathrm{N}-\mathrm{CH}_{3}\right), 4.18\left(\mathrm{q}, J=6.0 \mathrm{~Hz}, 2 \mathrm{H}, \mathrm{NHCH}_{2} \mathrm{CH}_{2} \mathrm{CH}_{3}\right)$, $8.05(\mathrm{~s}, 1 \mathrm{H}, \mathrm{CH}), 11.63(\mathrm{t}, J=6.0 \mathrm{~Hz}, 1 \mathrm{H}$, $\mathrm{N} H \mathrm{CH}_{2} \mathrm{CH}_{2} \mathrm{CH}_{3}$ ), 13.60 (br s, $1 \mathrm{H}$, Boc-NH).

${ }^{13} \mathrm{C} \mathrm{NMR}\left(\mathrm{CDCl}_{3}\right): \delta=11.82\left(\mathrm{NHCH}_{2} \mathrm{CH}_{2} \mathrm{CH}_{3}\right), 14.7$ $\left(\mathrm{C}-\mathrm{CH}_{3}\right), \quad 23.49 \quad\left(\mathrm{NHCH}_{2} \mathrm{CH}_{2} \mathrm{CH}_{3}\right), \quad 28.57 \quad(\mathrm{O}-$ $\left.\mathrm{C}\left(\mathrm{CH}_{3}\right)_{3}\right), 29.00\left(\mathrm{~N}-\mathrm{CH}_{3}\right), 47.57\left(\mathrm{NHCH}_{2} \mathrm{CH}_{2} \mathrm{CH}_{3}\right)$, $80.24\left(\left(\mathrm{CH}_{3}\right)_{3}-\mathrm{C}-\mathrm{O}\right), 98.04$ (C8a), 120.13 (C1a), 142.97 (C3a), 148.94 (C2), 149.19 (C9), 151.78 (C4a), 156.50 (C6), 160.73 (HNCOO), 163.65 (C8).

(9-Butylamino-2,3-dimethyl-3H-1,3,4,5,7-pentaazacyclopenta $[b]$ naphthalen-8-yl)- carbamic acid tertbutyl ester 28
Using the procedure described for compound 27, compound $\mathbf{2 8}$ was obtained from (9-methanesulfonyl2,3-dimethyl-3H-1,3,4,5,7-pentaaza-

cyclopenta[$[b]$ naphthalen-8-yl)-carbamic acid tert-

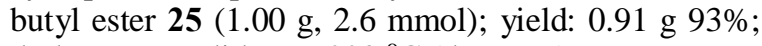
dark orange solid; mp $220{ }^{\circ} \mathrm{C}$ (decomp.).

IR (KBr): 1041, 1157, 1264, 1367, 1585, 1700, 1718, 1621, 1646, 2867, 2924, 2964, $3439 \mathrm{~cm}^{-1}$.

${ }^{1} \mathrm{H}$ NMR $\left(\mathrm{CDCl}_{3}\right): \delta=0.99(\mathrm{t}, J=7.0 \mathrm{~Hz}, 3 \mathrm{H}$, $\left.\mathrm{NHCH}_{2} \mathrm{CH}_{2} \mathrm{CH}_{2} \mathrm{CH}_{3}\right), 1.52\left(\mathrm{~s}, 9 \mathrm{H}, \mathrm{C}\left(\mathrm{CH}_{3}\right)_{3}\right)$, 1.62$1.76 \quad\left(\mathrm{~m}, \quad 4 \mathrm{H}, \quad \mathrm{NHCH}_{2} \mathrm{CH}_{2} \mathrm{CH}_{2} \mathrm{CH}_{3}\right.$ and $\left.\mathrm{NHCH}_{2} \mathrm{CH}_{2} \mathrm{CH}_{2} \mathrm{CH}_{3}\right), 2.54$ (s, 3H, C-CH $), 3.72$ (s, $\left.3 \mathrm{H}, \quad \mathrm{N}-\mathrm{CH}_{3}\right), \quad 4.21$ (q, $J=6.0 \mathrm{~Hz}, 2 \mathrm{H}$, $\mathrm{NHCH}_{2} \mathrm{CH}_{2} \mathrm{CH}_{2} \mathrm{CH}_{3}$ ), 8.04 (s, 1H, CH), 11.58 (br s, $1 \mathrm{H}, \mathrm{NHCH}_{2} \mathrm{CH}_{2} \mathrm{CH}_{2} \mathrm{CH}_{3}$ ), 13.62 (br s, $1 \mathrm{H}$, Boc-NH).

${ }^{13} \mathrm{C} \mathrm{NMR}\left(\mathrm{CDCl}_{3}\right): \delta=14.40\left(\mathrm{NHCH}_{2} \mathrm{CH}_{2} \mathrm{CH}_{2} \mathrm{CH}_{3}\right)$, $14.68\left(\mathrm{C}-\mathrm{CH}_{3}\right), 20.29\left(\mathrm{NHCH}_{2} \mathrm{CH}_{2} \mathrm{CH}_{2} \mathrm{CH}_{3}\right), 28.60$ $\left(\mathrm{O}-\mathrm{C}\left(\mathrm{CH}_{3}\right)_{3}\right), \quad 29.00 \quad\left(\mathrm{~N}-\mathrm{CH}_{3}\right), \quad 32.28$ $\left(\mathrm{NHCH}_{2} \mathrm{CH}_{2} \mathrm{CH}_{2} \mathrm{CH}_{3}\right) 45.54\left(\mathrm{NHCH}_{2} \mathrm{CH}_{2} \mathrm{CH}_{2} \mathrm{CH}_{3}\right)$, $80.22\left(\left(\mathrm{CH}_{3}\right)_{3}-\mathrm{C}-\mathrm{O}\right), 98.02$ (C8a), 120.13 (C1a), 143.00 (C3a), 148.91 (C2), 149.20 (C9), 151.77 (C4a), 156.50 (C6), 160.74 (HNCOO), 163.68 (C8).

\section{5-Amino-4-(1-methylsulfanyl-2,2-dicyanovinyl)-1- (2'-deoxy-3',5'-di-O-p-toluoyl- $\beta$-D-ribofuranosyl) imidazole 30}

1-(2'-Deoxy-3',5'-di- $O$ - $p$-toluoyl- $\beta$-D-erythropentofuranosyl)-5-nitroimidazole $\mathbf{2 9}^{99}(3.00 \mathrm{~g}, 6.4$ $\mathrm{mmol})$, and $5 \% \mathrm{Pd} / \mathrm{C}$ catalyst $(3.00 \mathrm{~g})$ were placed in dry THF (120 mL) and the mixture was hydrogenated at $\mathrm{rt}$ and atmospheric pressure, with vigorous stirring $(2 \mathrm{~h})$. The solution was filtered through dry celite, under argon, into a flask containing 2-(bismethylsulfanyl-methylene)-malononitrile 9 (5.50 g, $32.3 \mathrm{mmol}$ ). The celite was washed with a further portion of THF $(120 \mathrm{~mL})$. The reaction was stirred at $50{ }^{\circ} \mathrm{C}$, under argon, overnight giving a dark brown solution. The mixture was evaporated to dryness and the residue treated with liquid nitrogen-chilled EtOAc (200 mL). The solution was then filtered to give the crude product which was purified by column chromatography eluting with $\mathrm{CH}_{2} \mathrm{Cl}_{2}$, to remove excess starting material, and then with $1 \% \mathrm{MeOH}$ in $\mathrm{CH}_{2} \mathrm{Cl}_{2}$. The relevant fractions were combined and evaporated to dryness to give the amine $\mathbf{3 0}(0.53 \mathrm{~g}, 15 \%)$ brown crystalline solid; $\mathrm{mp} 98-100{ }^{\circ} \mathrm{C}$.

IR (KBr): 752, 1102, 1178, 1209, 1270, 1311, 1377, $1449,1486,1508,1544,1578,1611,1720,2209$, $2923,3035,3329 \mathrm{~cm}^{-1}$.

${ }^{1} \mathrm{H}$ NMR $\left(\mathrm{d}_{6}\right.$-DMSO): $\delta=2.30\left(\mathrm{~s}, 3 \mathrm{H}, \mathrm{S}-\mathrm{CH}_{3}\right), 2.39$ (s, 3H, Ar-CH $), 2.41\left(\mathrm{~s}, 3 \mathrm{H}, \mathrm{Ar}-\mathrm{CH}_{3}\right), 2.70(\mathrm{~m}, 1 \mathrm{H}$, 2ôCH), $2.88(\mathrm{~m}, 1 \mathrm{H}, 2 \hat{o} \mathrm{CH}), 4.54\left(\mathrm{~m}, 3 \mathrm{H}, 5 \hat{o} \mathrm{CH}_{2}\right.$ and 4ôCH), $5.63(\mathrm{~d}, J=4.0 \mathrm{~Hz}, 1 \mathrm{H}, 3 \hat{\mathrm{o} C H}), 6.19(\mathrm{~m}$, 1H, 1ôC $H$ ), 7.18 (br s, 2H, NH$\left.H_{2}\right), 7.34$ (d, $J=8.0 \mathrm{~Hz}$, $2 \times 2 \mathrm{H}, \mathrm{Ar}-H), 7.38(\mathrm{~d}, J=8.0 \mathrm{~Hz}, 2 \mathrm{H}, 2 \times \mathrm{Ar}-H)$, 7.79 (s, 1H, imidazole(2)- $H$ ), 7.88 (d, $J=8.0 \mathrm{~Hz}, 2 \mathrm{H}$, 2 x Ar- $H$ ), 7.97 (d, $J=8.0 \mathrm{~Hz}, 2 \mathrm{H}, 2$ x Ar- $H$ ). 
${ }^{13} \mathrm{C}$ NMR (d 6 -DMSO): $\delta=17.61\left(\mathrm{~S}-\mathrm{CH}_{3}\right), 21.09(2 \mathrm{x}$ $\mathrm{Ar}-\mathrm{CH}_{3}$ ), 36.08 (C20̂), 64.02 (C5ô), 64.40 (C3'), 74.80 $\left(C(\mathrm{CN})_{2}\right), 81.59(C 10 \hat{)}, 82.73(C 4 \hat{o}, 114.16(C 4-$ imidazole), $126.41(2 \mathrm{x} C \mathrm{~N}), 129.22$ (2 x $\mathrm{ArC})$, 129.30 (4 x ArC), 129.45 (4 x ArC), 132.29 (C2imidazole), $143.82\left(C-\mathrm{CH}_{3}\right), 144.04\left(C-\mathrm{CH}_{3}\right), 145.42$ (C5-imidazole), $165.08(C=\mathrm{O}), 165.38(C=\mathrm{O}), 167.13$ $\left(\boldsymbol{C}=\mathrm{C}(\mathrm{CN})_{2}\right)$.

MS (EI): $m / z(\%)=558(5)\left[\mathrm{M}+\mathrm{H}^{+}\right], 234(52), 219$ (10), 179 (13), 154 (43), 153 (15), 119 (27), 115 (58), 98 (100), 81 (85).

Anal. Calcd. for $\mathrm{C}_{29} \mathrm{H}_{27} \mathrm{~N}_{5} \mathrm{O}_{5} \mathrm{~S}$ (557.62): C, 62.47; H, 4.88; N, 12.56 .

Found: C, 62.30; H, 4.65, N, 12.26.

\section{Acknowledgment}

We thank the EPSRC for a studentship (to JAC) and the EPSRC National Mass Spectrometry Service for mass spectra.

\section{References}

(1) Leonard, N. J.; Sprecker, M. A.; Morrice, A. G. J. Am. Chem. Soc., 1976, 98, 3987.

(2) Scopes, D. I.; Barrio, J. R.; Leonard, N. J. Science, 1977, 195, 296.

(3) Leonard, N. J.; Keyser, G. E. Proc. Nat. Acad. Sci. USA, 1979, 76, 4262.

(4) Leonard, N. J. Acc. Chem. Res., 1982, 15, 128.

(5) Lessor, R. A.; Gibson, K. J.; Leonard, N. J. Biochemistry, 1984, 23, 3868.

(6) Seley, K. L.; Januszczyk, P.; Hagos, A.; Zhang, L.; Dransfield, D. T. J. Med. Chem., 2000, 43, 4877.

(7) Minakawa, N.; Kojima, N.; Hikishama, S.; Sasaki, T.; Kiyosue, A.; Atsumi, N,; Ueno, Y.; Matsuda, A. J. Am. Chem. Soc., 2003, 125, 9970.
(8) Liu, H.; Gao, J.; Maynard, L.; Saito, Y. D.; Kool, E. T. J. Am. Chem. Soc., 2004, 126, 1102.

(9) Krueger, A. T.; Lu, H.; Lee, A. H. F.; Kool, E. T. Acc. Chem. Res., 2007, 40, 141.

(10) Humphries, M. J.; Ramsden, C. A. Synthesis, 1999, 985.

(11) Clayton, R.; Davis, M. L.; Fraser, W.; Li, W.; Ramsden, C. A. Synlett, 2002, 1483.

(12) Davis, M. L.; Fraser, W.; Ramsden, C. A. manuscript in preparation.

(13) Al-Shaar, A. H. M.; Gilmour, D. W.; Lythgoe, D. J.; McClenaghan, I.; Ramsden, C. A. J. Chem. Soc. Perkin Trans. 1, 1992, 2779.

(14) Al-Shaar, A. H. M.; Chambers, R. K.; Gilmour, D. W.; Lythgoe, D. J.; McClenaghan, I.; Ramsden, C. A. J. Chem. Soc. Perkin Trans. 1, 1992, 2789.

(15) Lythgoe, D. J.; Ramsden, C. A. Adv. Heterocyl. Chem., 1994, 61,1 .

(16) Mashraqui, S. H.; Hariharasubrahmanian, H. J. Chem Res., 1999, 8, 492.

(17) Dewar, M. J. S.; Zoebisch, E. G.; Healy, E. F.; Stewart, J. J. P. J. Am. Chem. Soc., 1985, 107, 3902.

(18) Finnerty, J.; Mitschke, U.; Wentrup, C. J. Org. Chem., 2002, 67, 1084 . 
Please place the graphical abstract and short title of the article here. The short title will be used as a running header. Manuscript submission checklist

ÅStatement of significance of work.

ÅFull mailing address, telephone and fax numbers, and email address of the corresponding author. ÅPaper save as a PDF file.

ÅOriginal Word file.

Åriginal graphic files.

ÅGraphical abstract. 